\title{
Iris Segmentation Using a Statistical Approach
}

\author{
Luis M. Zamudio-Fuentes ${ }^{1}$, Mireya S. García-Vázquez ${ }^{1}$, \\ and Alejandro A. Ramírez-Acosta ${ }^{2}$ \\ ${ }^{1}$ Centro de Investigación y Desarrollo de Tecnología Digital (CITEDI-IPN), \\ Avenida del Parque 1310, Tijuana, B.C. México 22510 \\ ${ }^{2}$ MIRAL. R\&D, 1047 Palm Garden, Imperial Beach, 91932 USA \\ \{zamudio,mgarciav\}@citedi.mx, ramacos10@hotmail.com
}

\begin{abstract}
Eyelashes and reflections occluding the iris region are noise factors that degrade the performance of iris recognition. If these factors are not eliminated in iris segmentation phase, they are incorrectly considered as the iris region. Thus, produce false iris pattern information which decreases the recognition rate. In this paper a statistical approach is used to improve iris segmentation phase eliminating this noise from none constrain images, which is composed in three parts, finding the pupil and limbus boundary, reflection detection and eyelash detection. First an edge map is calculated using canny filter then the Circular Hough Transform is used to improve circle parameter finding. An intensity variation analysis is use to recognize a strong reflection. Eyelashes are classified in two categories, separable and multiple. Intensity variances are used to detect multiple eyelashes and an edge detector to localize separable eyelashes. The results show that statistics are useful to decide when is necessary applied the eyelash detector.
\end{abstract}

Keywords: Iris recognition, biometric, segmentation, eyelash detector.

\section{Introduction}

Iris recognition, is the most reliable biometric in terms of recognition and identification performance [1]. However, the performance of these systems is affected by inaccuracy segmentation $[2,3]$. Indeed, the false iris region information decreases the recognition rate [4, 5 and 6]. It is therefore importance to identify the source of noise such as eyelashes and reflections for improving the quality of the segmentation and then the performance of the iris recognition. In the previous iris segmentation approaches $[7,8]$ this noise is not considered, just the inner and outer boundary of an iris is founded. The aim of this paper is to improve iris segmentation method using a statistical approach and decide when eyelashes and reflection detection is necessary to remove these noise from the image and improve the accuracy of the iris recognition.

The remainder of this paper is organized as follows. Section 2 reviews the reflection detection; in section 3 and 4 discuss inner and outer boundary detection of an iris and the eyelash detection model, respectively. Implementation, results and discussion are presented in section 5. Finally, in section 6, we draw discussion and give suggestions for future work. 


\section{Reflection Detection}

Iris recognition system needs to avoid reflection that could degrade the recognition performance. However, strong reflection may be present if the illumination is not adapted or the subject uses contact lenses, also some jewelry can reflect in to the eye too. In this case, the value of intensity of a pixel with a strong reflection should be larger than a certain threshold [6]. A strong reflection can be recognized by a simple inequality, $f(x, y)>K_{1}$, where $f(x, y)$ is a pixel in an image and $K_{1}$ is taken as 180 , used in the following experiment after an histogram analysis of the grey scale intensity of the pixels.

\section{Inner and Outer Boundary Detection of an Iris}

Eye image contains pupil, iris, eyelids, eyelashes, sclera regions. However, for iris recognition the only area of interest is the iris region which has the patterns that are reported to remain unchanged over a life time [1], and they cannot be easily forged or modified. These patterns are delimited by the inner and outer boundary. The inner boundary is the circle which delimits de pupil and the iris region. On the other hand, outer boundary delimits the iris and the sclera. These two circles can be taken as two non concentric circles [9]. To define the each boundary and extract the iris region; the first step is to conduct edge detection to get the edge map of the eye images using a canny filter. The second step is to perform the circular Hough transform (CTH) [10]. The CTH is a "voting based" computational algorithm. For each boundary is necessary to obtain the coordinates of the center of the circle and the radius length. The CHT is used to transform a set of feature points in the image space into a set of accumulated votes in a parameter space. Then, for each feature point, votes are accumulated in an accumulator array for all parameter combinations. The array elements that contain the highest number of votes indicate the radius length and the coordinates of the center. The Circular Hough Transform [11, 12] has been implemented as follows:

1. An binarized edge map is calculated using a canny filter

2. Count each pixel in an edge map and obtain its position $\left(x_{i}, y_{i}\right)$, where $i$ is the total pixels in an edge map.

3. Set the radius range to find the boundary of interest.

4. Compute Circular Hough Transform.

for pixeledge $=1$ to $i$.

for $\mathrm{r}=1$ to maximum radius wanted

for $\mathrm{y}=1$ to maximum row in an edge map

Compute $x_{k}=\sqrt{r_{j}^{2}-\left(y-y_{i}\right)^{2}}+x_{i}$

$\mathrm{x}_{\mathrm{k}}$ represents the possible coordinate $\mathrm{x}$ of the center of the circle, $\mathrm{r}$ is the radius, $y$ is the row in an edge map, $y_{i}$ and $x_{i}$ are the positions of a pixel in an edge map.

After $x_{k}$ is calculated the accumulator array is increased as follows $\operatorname{ACC}\left(\mathrm{y}, \mathrm{x}_{\mathrm{k}}, \mathrm{r}_{\mathrm{j}}\right)=\operatorname{ACC}\left(\mathrm{y}, \mathrm{x}_{\mathrm{k}}, \mathrm{r}_{\mathrm{j}}\right)+1$

end all loops. 
5. Obtain the maximum value in the accumulator array to get $(\mathrm{x}, \mathrm{y})$ coordinates which belong to the center and radius length $\mathrm{r}$ of the circle of interest.

6. Repeat from step 3 and modify the radius range to obtain the outer boundary

This algorithm requires a radius range where the radius of interest could be located and the position of the pixels which belong to an edge map. First, the inner boundary is found; secondly, the algorithm finds the outer boundary. For each position in an edge map this algorithm generates a circle for each value in the radius range. The point where the majority of the circles intersect, that point will be the center of the boundary of interest.

\section{Eyelash Segmentation}

Two classes of eyelashes are defined, separable and multiple eyelashes. Separable eyelashes are defined as the eyelashes that can be distinguished from other eyelashes and multiple eyelashes are the eyelashes that overlap in a small area [6].

\subsection{Separable Eyelashes}

Separable eyelashes can be distinguished from other eyelashes [4]; the pixels around separable eyelashes should not belong to others. Because of the intensity difference between iris pixels and eyelash pixels, a separable eyelash can be regarded as an edge in an image. Base on this property, a real part of Gabor Filter [13, 14, 15] is proposed to detect separable eyelashes, which, is in the spatial domain has the following general form.

$$
G(x, u, \sigma)=e^{x^{2} / 2 \sigma^{2}} \cos (2 \pi u x)
$$

Where the frequency of the sinusoidal wave is $u$, the standard deviation of the Gaussian envelope is $\sigma$ and the parametric component which represent the real part of the Gabor filter is $\mathrm{x}$. In fact, the filter works as an edge detector. If the resultant value of a point is smaller than a threshold, it is noted that this point belong an eyelash.

This approach has been implemented by the follow algorithm.

1. Gabor filter is applied after strong reflection detection (cf. section 2) and iris localization (cf. section 3).

2. Compute the gradient direction and quantify the result in four angles 0,45 , 90, 135 degrees.

3. Set the frequency of the sinusoidal $u=1 / 4 \pi$. $1 / 2 \pi, 3 / 4 \pi$.

4. Calculate component $\mathrm{x}$.

5. Obtain Gabor filter coefficients using equation (1).

6. Spatially convolve image with the filter to get the enhanced image.

7. Eliminate the pixels which belong to an eyelash.

This algorithm, use Gabor filter to enhance the pixels and eliminates the separable eyelash. The filter is divided in different frequency scales, to ensure that the same proportion of the spectrum is covered in both dimensions. In this experiment three 
different frequencies are used. The first frequency used was $1 / 4 \pi$, then $1 / 2 \pi$ and $3 / 4 \pi$. The result is a set of filters [15] that covers one half of the frequency plane, the other half of the plane is not needed because the extra filters would have the same response as the existing ones.

\subsection{Multiple Eyelashes}

Many eyelashes overlap in a small area. Such that, the change of intensity variation in this area is almost zero. Thus, is necessary to obtain the variance of the intensity in this area and verify if is smaller than a threshold. It can be described [6] as:

$$
\sum_{i=-N}^{N} \sum_{j=-N}^{N} \frac{(f(x+i, y+j)-M)^{2}}{(2 N+1)^{2}}<K_{2}
$$

Where $\mathrm{M}$ is the mean of intensity in the small window; $(2 \mathrm{~N}+1)^{2}$ is the window size and $\mathrm{K}_{2}$ is a threshold.

\section{Implementation and Results}

In the experiments we test the segmentation system with 150 images from the MBGC NIR eyes still data base [16]. This contains 8590 eyes images. In this data set were acquired using an Iridian LG EOU 2200 camera.

The figure 1 shows a diagram with all the steps to obtain accurate iris segmentation. Once the eye image is obtained, the first step consist in verify if it has a strong reflection (cf. section 2). Then an edge map is calculated using canny filter to improve circle parameter finding. We use CHT to calculate iris/pupil circle parameter.

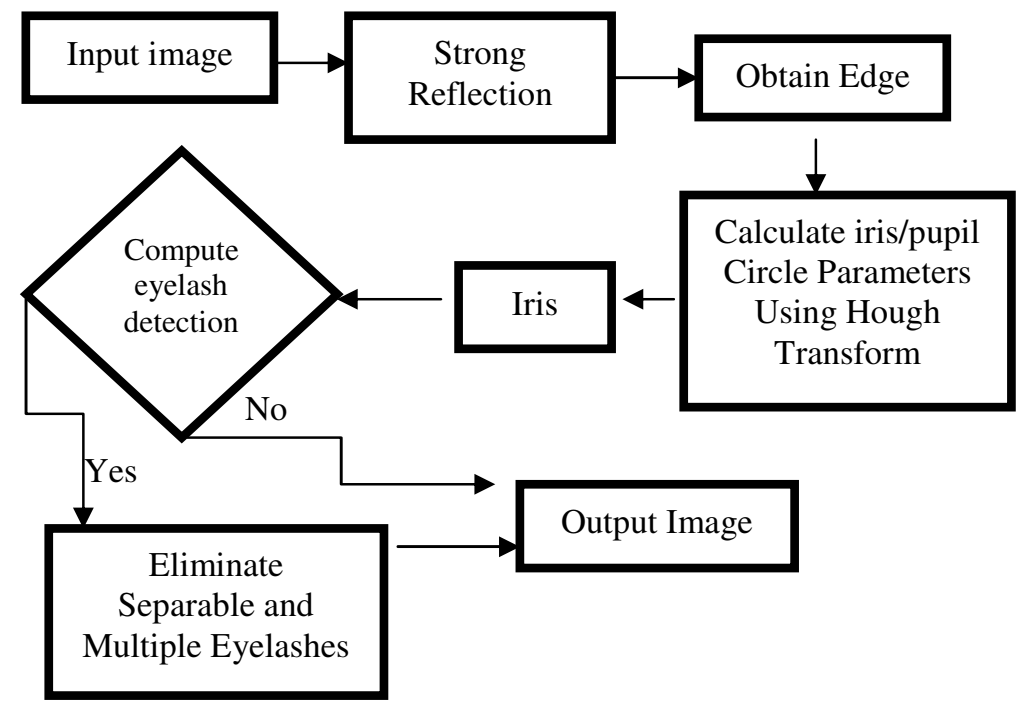

Fig. 1. All steps to obtain accurate iris segmentation 
The algorithm uses these parameters to extract the iris region (cf. section 3). However, after eye data base analysis, it shows that it is more probable to find eyelash just in the region near at the upper eyelid or lower eyelid than the region where the pupil exist. Thus it is necessary to decide when the Gabor filter should be used. To resolve this issue, this algorithm divides the extracted iris region in three blocks (upper, middle and lower).

After extracted iris region is divided, the algorithm calculates the mean $\mu_{\mathrm{r}}$ and the standard deviation $\sigma_{\mathrm{r}}$ of the whole region. Then the algorithm calculates the mean $\mu \mathrm{l}_{\mathrm{j}}$ and the standard deviation $\sigma \mathrm{l}_{\mathrm{j}}$ for each $\mathrm{j}$ block. To determine which block has eyelash occlusion is important to analyze the intensity of the pixels. Due to changes of intensities, we conclude that: if one block has eyelashes the difference between the pixels is significant. Such that, its mean and standard deviation are bigger than the statistical values of the whole region (condition 3) then the eyelash segmentation algorithm is computed.

$$
\mu_{\mathrm{r}}<\mu \mathrm{l}_{\mathrm{j}} \text { and } \sigma_{\mathrm{r}}<\sigma \mathrm{l}_{\mathrm{j}}
$$

As a result we eliminated the eyelashes from the iris region without changing the original iris pattern. In other words, after removing the eyelashes region we obtain an accurate iris pattern free of any noise either from reflection or eyelash occlusion which will improve iris recognition performance. On the other hand, we compare our segmentation method versus Libor's Masek segmentation and eyelash detection method [17]. This algorithm eliminated the eyelashes by occluding them through a rectangle. The results show that our segmentation is accurate, it performed eyelash detection and it is $90 \%$ faster than Libor Masek. Some examples are shown in figure 2 which is distributed at the left row, is the original image, in the center row is the iris extracted from our algorithm and the right row is the iris extracted using Libor's method. Where images a) and b) shows that our algorithm finds the region of the eyelashes. The eyelashes issue increases when they are coated; due to the similitude of intensities between the pupil and eyelash pixels. From the image c) the algorithm could extract the iris region. The image d) shows the accuracy of our iris segmentation. However, Libor's method does not have that accuracy for this image. The Circular Hough Transform is the most intensive search as remarked in [8]. Even though, this algorithm is our first approach to iris recognition. This segmentation was
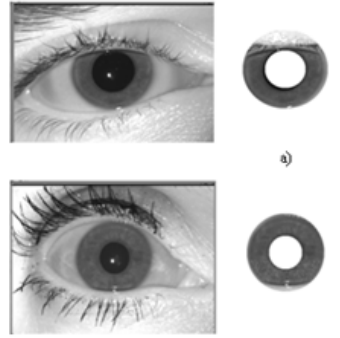

a)

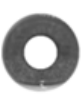

c)
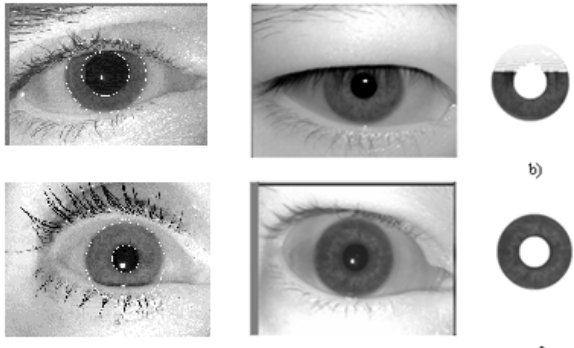

d)

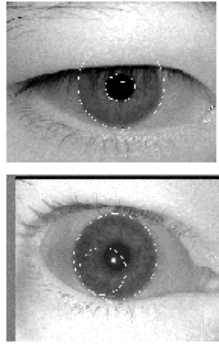

Fig. 2. Results of Iris image segmentation 
accurate and fast enough to keep working on it. However, computing time of the CHT is one limitation that will be improved in further work.

Our eyelash detection is base on [4]. On the other hand, we introduce the three iris region blocks to statistical study of intensity variation of the pixels which improve the eyelash detection.

\section{Discussions and Further Work}

We have shown how to extract the iris region, eliminate the reflections and remove the eyelash occlusion using statistical values. Our method is fast, straightforward to implement, and accurately for a wide variety of images from MBGC NIR eye still data base. This method explains how a Circular Hough Transform should be implemented. On one hand, after analyze the data base; this article introduces a new way to detect where the eyelashes are by dividing the iris region in three iris blocks (upper, middle and lower). Then each block is statistically evaluated and classified if that block has or not eyelashes to detect eyelash. For each detected block, this method analyzes the Gabor filter that is used to eliminate the separable eyelashes and study how much is the chance of intensity variation to eliminate multiple eyelashes. Nevertheless, our method does have some limitations, and there are several avenues for future work.

The primary limitation of our method is that computed time to find the pupil's search region be improved. The first further work will perform a histogram analysis combined with morphologic models as thinning to enhance pupil and iris segmentation. When the pupil's radius is too small close to ten pixels the segmentation method could not be accurate because the Circular Hough Transform is a voting method to find circles boundary. Where in an edge map each pixel votes. If some edge is bigger than a small pupil edge then other circle boundary will be founded instead of the pupil boundary. Other further work is that we will improve our method to analyze iris segmentation from video using the MBGC NIR eye video data base instead of the MBGC NIR eye still data base.

Acknowledgment. This work was supported by IPN-SIP20100030.

\section{References}

1. Daugman, J.: Results from 200 Billion iris Cross-Comparisons. Technical Report. UCAMCL-TR-635, ISSN 1476-2986, Number 635 (June 2005)

2. Zamudio, L.M., García, M.S., Colores, J.M.: Revisión de las Etapas de Adquisición y PreProcesamiento de Imagen en un Sistema de Reconocimiento Basado en Iris. VI TallerEscuela de Procesamiento de Imágenes PI09 CIMAT. Agosto, Gto. México (2009)

3. Xu, G.Z., Zhang, Z.F., Ma, Y.D.: Automatic Iris Segmentation Base on Local Areas. In: The 18th International Conference on Pattern Recognition (ICPR'06). IEEE, Los Alamitos (2006)

4. Kong, Z.: Detecting Eyelash and Reflection For Accurate Iris Segmentation. International Journal of Pattern Recognition and Artificial Intelligence 17(6), 1025-1034 (2003) 
5. Kang, P.: A Robust Eyelash Detection Based on Iris Focus Assessment. Pattern Recognition Letters 28, 1630-1639 (2007)

6. Yuan, W.H.: A Novel Eyelash Detection Method for Iris Recognition. In: Proceeding on the 2005 IEEE. Engineering in Medicine and Biology 27th Annual Conference Shanghai, China, pp. 6536-6539 (2005)

7. Lim, S., Lee, K., Kim, J.: Efficient iris recognition by characterizing key local variations. IEEE Transactions on Image Processing 13(6) (June 2004)

8. Otero-Mateo, N., Vega-Rodríguez, M.Á., Gómez-Pulido, J.A., Sánchez-Pérez, J.M.: A Fast and Robust Iris Segmentation Method. In: Martí, J., Benedí, J.M., Mendonça, A.M., Serrat, J. (eds.) IbPRIA 2007. LNCS, vol. 4478, pp. 162-169. Springer, Heidelberg (2007)

9. Chen, Y., Wang, J., Han, C., Wang, L., Adjouadi, M.: A robust segmentation approach to iris recognition based on video. In: 37th IEEE Applied Imagery Pattern Recognition Workshop, pp. 1-8 (2008)

10. Rizon, M., Yazid, H., Saad, P., Shakaff, A.Y.M., Sugisaka, A.R.S.M., Yaacob, S., Rozailan Mamat, M., Karthigayan, M.: Object Detection using Circular Hough Transform. American Journal of Applied Sciences 2(12), 1606-1609 (2005) ISSN 1546-9239

11. Hough Transform

http: / /www.vislab.uq.edu.au/education/sc3/2000/hough/report/ node 3. html

12. Hough Tranform

http://www.cis.rit.edu/class/simg782/lectures/lecture_10/ lec782_05_10.pdf

13. Gabor filter. Multiresolution Design of Multiple Gabor Filters for Texture Segmentation, http: / / wws 2 uncc. edu/tpw/diss/diss.html

14. Kovesi, P.: Gabor filter and Log-Gabor filter http: / /www.csse.uwa. edu.au/ pk/research/matlabfns/PhaseCongr uency / Docs / convexpl . html

15. Christmas, B.: Designing complex Gabor Filters (November 16, 2007), http: / /www.ee.surrey.ac.uk/CVSSP/Ravl/RavlDoc/share/doc/ RAVL/html/Gabor.pdf

16. Multi Biometric Grand Challenge MBGC, http: / / face.nist.gov/mbgc/

17. Masek, L., Kovesi, P.: MATLAB Source Code for a Biometric Identification System Based on Iris Patterns. The School of Computer Science and Software Engineering. The University of Western Australia (2003) 